\title{
Kinetic and thermodynamic modelling of the von Willebrand factor mediated bonds during platelet adhesion under flow conditions
}

\author{
M. Machin ${ }^{1}$, A. Santomaso ${ }^{1}$, M. Mazzucato ${ }^{2} \&$ P. Canu ${ }^{1}$ \\ ${ }^{I}$ Department of Chemical Engineering Principles and Practice (DIPIC), \\ University of Padova, Padova, Italy \\ ${ }^{2}$ Servizio Immunotrasfusionale e Analisi Cliniche C.R.O.-I.R.C.C.S., \\ Aviano (PN), Italy
}

\begin{abstract}
Platelet tethering to injured vascular surfaces exposed to rapidly flowing blood relies on the interaction with the adhesive protein von Willebrand factor (VWF). By perfusing blood through a flow chamber and analyzing surface events using a tailored/specific image processing and particle tracking algorithms, we derived non-conventional information, such as deposition and removal rates, supporting kinetics and thermodynamics speculations. Statistical reasoning allowed the formulation of single-event observations into mean-field behaviour, suitable for a macroscopic, clinical scale application. The arrest and release frequencies were quantified through association and dissociation rate constants, $k_{\text {on }}$ and $k_{\text {off }}$ Variables influencing the arrival/removal rate were identified and their dependence on different experimental factors is illustrated in detail. It is shown how variation of hydrodynamic forces expressed as wall shear rate can be used to sample adhesion rate and bond resistance, ultimately resulting in different $k_{\text {on/off }}$ values and equilibrium adhesion constant. The issue of establishing whether multiple bonds are synergistic in opposing tensile stress is addressed. Potentials, applicability and limitations of the proposed characterization of platelet adhesion to VWF-coated surfaces are discussed. Developments in this area of research can considerably help to elucidate important aspects of normal hemostasis and pathological arterial thrombosis.
\end{abstract}

Keywords: platelets, kinetics, association and dissociation rate constant, residence time, thermodynamics, adhesion equilibrium constant, image analysis. 


\section{Introduction}

Cell adhesion is a process of prominent biological importance. It is usually mediated by the interaction of dedicated receptor and ligand molecules bound to interacting surfaces. It is, therefore, of obvious interest to develop suitable methods for predicting whether a cell-to-cell encounter will result in durable adhesion. The formation of the normal haemostatic plug at sites of vascular injury (hemostasis) or the development of pathological thrombi on atheromatous plaque or onto an artificial biomaterial in a blood-contacting device (thrombosis) is critically dependent on the outcome of platelet adhesion (Ruggeri [1]). The ability of platelets to tether to injured vascular surfaces exposed to rapidly flowing blood relies on the interaction between the platelet glycoprotein receptor Ib $\alpha$ (GP Ib $\alpha$ ) and the adhesive protein von Willebrand factor (VWF) (Mendolicchio and Ruggeri [2]). Rates of adhesion and detachment are generally accepted as key determinants of cell adhesion mediated by receptor-ligand interactions, especially in a dynamic flow environment (Zhu [3]). Critical to the role of the initial bonds platelets-VWF is the balance of their on and off binding rates which must be tuned to support platelet tethering and transient arrests without the need to establish stable bonds (Savage et al. [4]). While an impressive amount of information has been gathered on the rate of bond dissociation (Doggett et al. [5]; Kumar et al. [6]), little attention has been given to the frequency of formation of molecular interactions, mainly because variables involved in adhesion rate cannot be easily determined. Progress in this direction has been made (Pierres et al. [7]), however a methodological framework for quantitative investigation of the parameters involved in cell-surface interaction (kinetics of the on and off process and characterization of the adhesion equilibrium) with specific attention to blood platelets is lacking. Fluorescence microscopy is used here to observe adhesion of platelets on a coverslip lined with VWF inserted on the lower surface of a rectangular perfusion chamber. With this study we aim at defining the experimental factors on whom the rate on and off are dependent, predicting their behaviour and elucidating how they can possibly be controlled. Thermodynamics considerations are also given.

\section{Materials and methods}

\subsection{Blood preparation}

Venous blood from healthy volunteers who were not taking any medication and who gave informed consent according to the Declaration of Helsinki was collected into syringes containing as anticoagulant the $\alpha$-thrombin inhibitor Dphenylalanyl-L-prolyl-L-arginine chloromethyl ketone dihydrochloride (PPACK; $46 \mu \mathrm{M}$ final concentration; Bachem, Bubendorf, Switzerland). Platelets were incubated for 20 minutes at $37^{\circ} \mathrm{C}$ with the calcium fluorescent probe fluo-3 acetoxymethyl ester-AM (Fluo 3-AM; $8 \mu \mathrm{M}$ final concentration; Molecular Probes, Eugene, OR), as previously described in detail (Mazzucato et al. [8]). 


\subsection{Flow experiments}

Human plasma VWF was diluted in phosphate-buffered saline (PBS; $20 \mathrm{mM}$ dibasic sodium phosphate, $\left.20 \mathrm{mM} \mathrm{NaH}{ }_{2} \mathrm{PO}_{4}, 2.7 \mathrm{mM} \mathrm{KCl}, 0.15 \mathrm{M} \mathrm{NaCl}\right), \mathrm{pH}$ 7.4. Different concentrations of protein were used to coat the glass coversilps ( 24 x $50 \mathrm{~mm}$ ) that represented the lower surface of a parallel plate flow chamber. A silicon rubber gasket determined the flow path height $(125 \mu \mathrm{m})$ between the glass coverslip and the upper plate. The chamber was assembled and filled with PBS, pH 7.4. A syringe pump (Harvard Apparatus Inc., Boston, MA) and silicone tubing connections were used to aspirate blood through the chamber at the desired flow rate. The perfusion system was mounted onto an inverted microscopes equipped with epifluorescent illumination (Diaphot-TMD; Nikon Instech, Kanagawa, Japan) and intensified CCD video camera (C-2400-87; Hamamatsu Photonics, Shizuoka, Japan). All these experiments were recorded at a video rate of $25 \mathrm{fps}$ on S-VHS videotape. Recorded images were captured and digitized from videotape. Image analysis was then performed off-line.

\subsection{Image processing technique}

Image analysis is crucial for the following theoretical discussion. It must provide reliable identification and tracking of cells on long image sequences, rapidly (i.e., without manual operations on individual images) and consistently. For that purpose we developed our own algorithms and programs (Machin [9]), based on the MATLAB Image Tool (The MathWorks, Inc. [10]). Shortly, original features of the method include options for automatic thresholding greyscale images, and dedicated tracking algorithms, able to account for the physics of the flow experiment. Object identification between subsequent frames is obtained with a time-varying, two dimensional probability density function build around each single identified object. Details can be found elsewhere (Machin et al. [9; 11]).

\section{Adhesion data analysis}

Continuous monitoring and appropriate quantitative treatment of the digitized images can unravel quantitative information on the establishment and breakage of adhesive bonds between a platelet moving in shear flow and the substrate, as well as the path travelled by the cell on the surface as a function of time. Thanks to the automatic particle tracking algorithm we developed, details on each particle movement can be collected. Fig. 1 illustrates a representative platelet instantaneous velocity along the flow direction, which shows that the motion on the surface is discontinuous with several 'stop and go' movements.

The platelet stays on the surface for a certain time, then roll on the surface, stops, starts rolling again, many times before final detachment, and the duration of each step may vary. The actual history of platelet displacements is decisive in understanding and quantifying the strength of adhesion, as well as characterizing the surface density of ligands. 


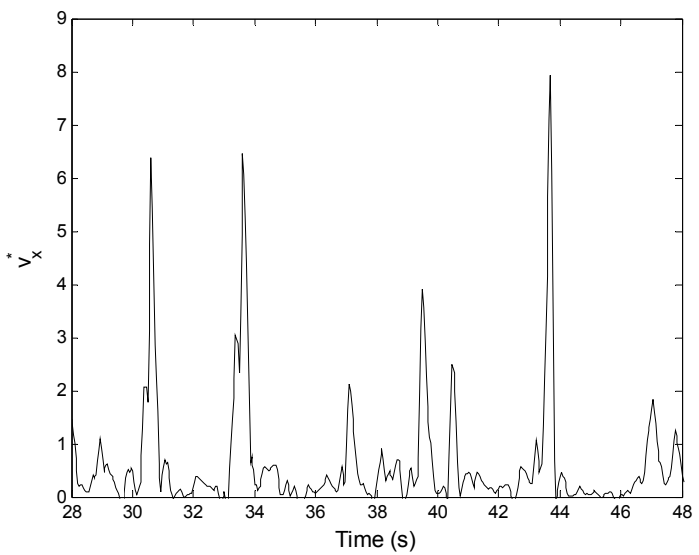

Figure 1: Instantaneous platelet velocity in the flow direction $(x)$ expressed as number of mean diameters per second along a glass surface coated with VWF used at saturation. The wall shear stress is $105 \mathrm{dyn} / \mathrm{cm}^{2}$.

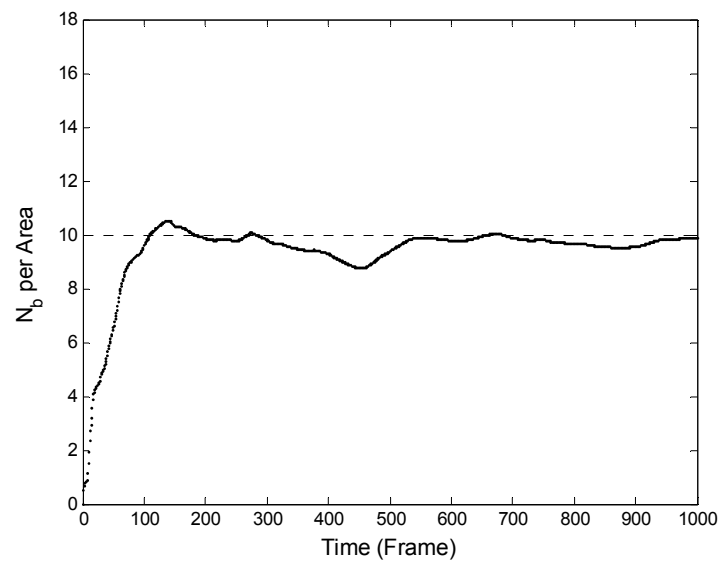

Figure 2: Progressive average of the number of platelets bound to the surface per unit area as a function of time. Asymptotic average is also indicated (dotted line).

Fig. 2 illustrates the variation with time of the number of platelet-substrate encounters $\left(N_{b}\right)$. After a transitory, the system reaches the adhesion equilibrium and the number of surface events remains fairly constant. The length of the initial transitory state indicates the minimum interval of observation required to collect statistically significant data. As suggested by Fig. 2, quite a long observation period (approximately 125 frames with a temporal resolution of 25 
fps) must be allowed to reliably identify the average surface concentration (equilibrium between the on and off process). On the other hand, the asymptotic value changes according to the experimental conditions such as wall shear stress, platelet count, hematocrit, and surface concentration of active sites. Note that varying a certain condition, for example the wall shear stress (through different flow rates imposed by the pump), often requires adjusting the observation time to have a significant value for the deposition/removal rates. All our experiments were recorded in steady-state conditions on the surface. Secondary data, directly determined from observables, can then be derived such as the distribution of lifetimes for all the interacting platelets.

Of specific interest for a kinetic and thermodynamic study is the direct measurement of on and off rates. Fig. 3 shows the cumulated number of platelets that tethered and detached in the field of view up to a given time. The slope of the curve represents the rate of attachment $\left(\mathrm{R}_{\text {on }}\right)$ and detachment $\left(\mathrm{R}_{\text {off }}\right)$.

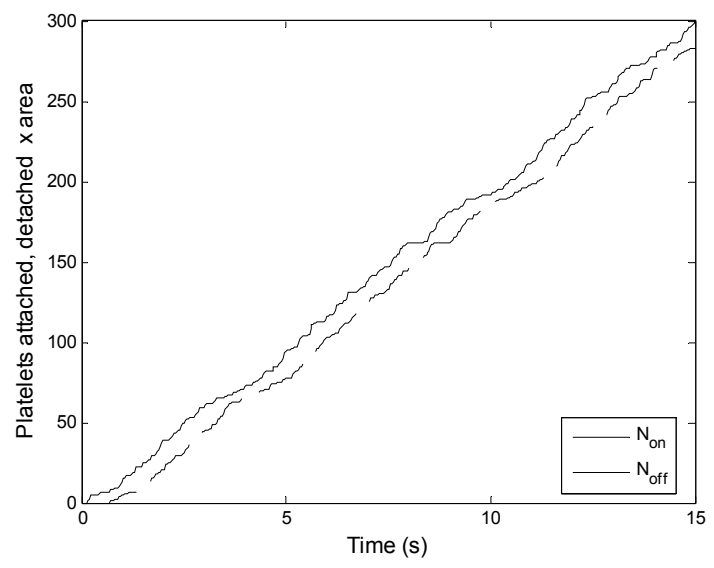

Figure 3: Cumulated number of platelets that tethered (solid line) and detached (dashed line) in the field of view as a function of time.

\subsection{Adhesion kinetics}

Bell's model (Bell [12]) is the classical reference on quantification of biological bond formation and rupture. According to Bell, the variation with time of the number of cells bound to the surface, $N_{b}$, is given by analogy with a reversible bimolecular chemical reaction between unbound cells $(u)$ and surface sites $(s)$ :

$$
u+s \underset{\text { off }}{\stackrel{o n}{\rightleftharpoons}} b
$$

so that

$$
\frac{d N_{b}}{d t}=R_{o n}-R_{o f f}=k_{o n} N_{s} N_{u}-k_{o f f} N_{b} .
$$

$k_{o n}$ and $k_{\text {off }}$ are the kinetics constant of the on and off process, respectively; $N_{u}$ is the number of unbound receptors on flowing platelets that can interact with the 
surface, and $N_{s}$ is the number of sites of VWF available for the platelets to bind. The frequency of arrival/removal rate is directly measurable as shown above (see Fig. 3). Evaluation of the intrinsic parameters ( $k$ 's) from measured rates requires some considerations on the quantities involved in eqn (2). The instantaneous and average number of platelets bound to the surface are experimentally measurable through continuous image analysis. The same measurements provide an estimate of $\mathrm{R}_{\text {off }}$, as shown in Fig. 3. Then, the off-rate kinetic constant $k_{\text {off }}$ is easily calculable as the ratio between $\mathrm{R}_{\text {off }}$ and the average number of bound platelets. Different experiments can reveal the dependence of $k_{\text {off }}$ on experimental factors, such as wall shear rate. Variables involved in the adhesion rate $\left(R_{o n}\right)$ are not as easily determined. The number of sites on the surface available for the platelets to bind can be expressed as the difference between total number of sites provided by the surface, $N_{s}^{o}$, and those already drawn in binding platelets

$$
N_{s}=N_{s}^{o}-\alpha N_{b} \quad N_{s}^{o}=K_{V W F} C_{V W F}
$$

$N_{s}^{o}$ can be related to the concentration of substrate (von Willebrand factor in our studies), $C_{V W F}$, used to coat the surface. The full adsorption curve which can be determined by binding assays experiments (see Fig. 4a in the following, by analogy) reduces to a simple proportionality at low substrate concentration before surface saturation begins. Slope of the adsorption curve in the linear region is given by the adsorption equilibrium constant, $K_{V W F}$. The coefficient $\alpha$ is the number of bonds a single platelet can establish with the surface ligands. It is frequently assumed $\alpha=1$, the minimum for platelet-surface interaction, although evidences of multiple bond formation (i.e., rolling on the surface) have been observed. Different $\alpha$-values can be tested to check whether multiple bonds are synergistic in opposing tensile stress. A procedure to estimate $\alpha$ will be given below. The platelets that can bind to the surface are only those in the marginal layer. However, the platelet concentration close to a surface has been demonstrated to be quite different from the blood platelet count, $N_{p}$, because of radial segregation of cells in blood (Aarts [13]). We can assume

$$
N_{u}=\beta \delta N_{P} .
$$

The parameter $\delta$ translates the average platelet count into the number concentration of platelet in the marginal layer; note that it can be (much) higher than 1. $\beta$ is the number of receptors per platelet that can be only guessed. Assembling all the above developments in eqn. (2), we can express the adhesion rate in term of measurable variables:

$$
\begin{aligned}
R_{o n} & =k_{o n}\left(K_{V W F} C_{V W F}-\alpha N_{b}\right) \cdot \beta \delta N_{p} \\
& =k_{o n}^{\prime}\left(K_{V W F} C_{V W F}-\alpha N_{b}\right) N_{p}
\end{aligned}
$$

Provided measurements of $R_{o n}$ and $N_{b}$ from image analysis of flow experiments, as shown in Figs. 2 and 3 above, the estimation of the actual adhesion kinetic constant $k_{\text {on }}$ requires knowledge of several additional data. Among them, platelet count $N_{p}$ and substrate concentration $C_{V W F}$ are normally known, substrate adhesion constant $K_{V W F}$ can be determined through binding assays, while $\alpha, \beta$, and $\delta$ factors are unknown. Interestingly, adhesion experiments can be used to 
assess $\alpha$ that represents the number of bonds truly formed on the surface by simply using measurement from at least two experiments with a different platelet count and/or substrate concentration. On the contrary, $\beta$ and $\delta$ factors remains undistinguishable from $k_{o n}$, so that an apparent adhesion kinetic constant, $k_{\text {on }}=\beta \delta k_{\text {on }}$ will be determined from measurements of $R_{o n}$. Unless techniques are identified to independently measure the actual platelet concentration in the marginal layer (i.e., $\delta$ ) and the number of receptors per platelet (i.e., $\beta$ ), we wont be able to estimate the intrinsic adhesion rate constant $k_{\text {on }}$ by measurements of the adhesion rate $R_{o n}$. Uncertainties are mostly in the determination of the platelet concentration at the surface which is heavily dependent on the fluid flow mechanics in specified vessel geometry, and the blood characteristics that can affect it, such as hematocrit and other variables that can influence its viscosity. We are not aware of any predictive model for radial platelet concentration at the present, so that estimation of pure $k_{\text {on }}$ remains only an attempt.
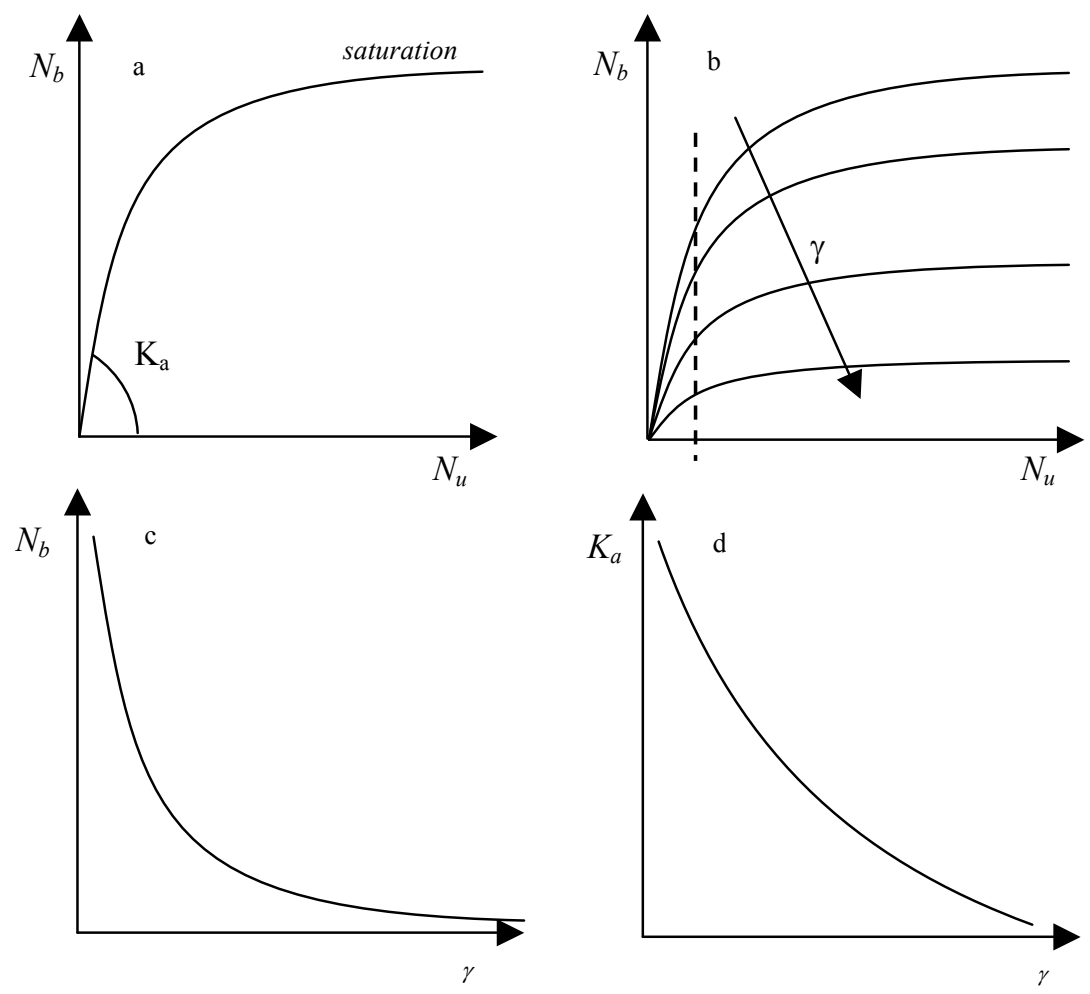

Figure 4: Thermodynamics of adhesion: a) bound versus unbound platelets at equilibrium; b) effect of wall shear rate on the equilibrium curves; non-saturation, constant platelet count conditions are marked by a vertical dotted line; c) effect of wall shear rate on the equilibrium surface concentration of platelets, and d) on the equilibrium constant. 


\subsection{Adhesion thermodynamics}

Thermodynamics aims at characterizing and predicting the equilibrium in a process. The adhesion-desorption of cells on a surface is said to be macroscopically in an equilibrium state when the rate of surface arrival is equal to the rate of departure from the surface:

$$
R_{o n}=R_{\text {off }} \quad \text { or } \quad \frac{d N_{b}}{d t}=0
$$

Under such circumstances we can build up the expression of the equilibrium surface concentration of cells by developing the expressions of the rates:

$$
R_{\text {on }}=k_{\text {on }} N_{s} N_{u}=k_{\text {off }} N_{b}=R_{\text {off }}
$$

It must be recalled that the surface concentration of available sites, $N_{s}$, is related to the total number of surface sites, $N_{s}^{o}$, reduced by those occupied by cells as in eqn. (3). Combining eqns (7) and (3) we obtain the equilibrium concentration:

$$
N_{b}=\frac{K_{a} N_{u} N_{s}^{o}}{1+\alpha K_{a} N_{u}} \quad K_{a} \equiv \frac{k_{o n}}{k_{\text {off }}}
$$

where the adhesion equilibrium constant $K_{a}$ has been defined. Eq. (8) has the typical Langmuir adsorption form, as shown in Fig. 4a.

The same curve applies to the substrate as observed in binding assay techniques, in static conditions, and provides the adhesion equilibrium constant $\left(K_{V W F}\right)$ used above. Flow experiments give a unique opportunity to investigate the strength of the adhesive bond. Increasing the flow rate, and then the wall shear stress, the amount of platelets bound to the surface as a function of unbound platelet concentrations is expected to decrease, as schematically shown in Fig. 4b. Experiments at different platelet count can be planned to identify the whole equilibrium curve, but it is more practical to perform experiments always at the same (approx.) platelet count. The effect of the wall shear stress on the equilibrium constant can be assessed anyway. For that purpose, non-saturation conditions (see Fig. 4b) are preferred since $K_{e q}$ variations can be directly measured. In such a case, the number of binding cells is expected to vary with wall shear rate, as shown in Fig $4 \mathrm{c}$ and consequently the equilibrium constant similarly, as in Fig. 4d. Note that a lower average concentration of platelets on the surface or a smaller equilibrium constant does not imply lower on- and offrates. On the contrary they are expected to increase, with the wall shear rate, while the equilibrium surface concentration decreases anyway. The function $K_{a}(\gamma)$, once experimentally measured, can be used to determine the dependence on wall shear rate of the individual kinetics constants, according to

$$
K_{a}(\gamma)=\frac{k_{o n}^{0}}{k_{o f f}^{0}} e^{\left(B_{o n}-B_{o f f}\right) \gamma}
$$

where $B$ 's are the compliance factors explaining the sensitivity of each individual constant to the shear rate. Unfortunately, the assumption of pure removal (neglect of arrival rate) is often used to determine $k_{\text {off }}$ so that the observed 
exponential dependence of the kinetic constants on the applied force (i.e., $\gamma$ ), can be misleading.

\section{Conclusions}

A method of adhesion data analysis, combining tailored image processing techniques, statistical analysis, kinetic and thermodynamics modelling has been proposed. The study is motivated by and has been applied to the initial interaction of platelets with surface-immobilized von Willebrand factor in flow experiments. Results indicate the key role of a reliable automatic image processing procedure to provide information critical in explaining how biomechanical properties of tether bonds ultimately control the process of platelet adhesion. Non-conventional information, such as deposition and removal rates could be obtained in a number of experimental conditions, supporting kinetics and thermodynamics speculations. The effort to develop an appropriate interpretation of adhesion data is a valuable contribution to help elucidating the adhesive properties of platelets that determine their participation in atherogenesis and ultimately the formation of occlusive thrombi. By understanding the multifaceted mechanism involved in platelet interactions with vascular surfaces, new approaches can be tailored to selectively inhibit the pathways most relevant to the pathological aspects of atherothrombosis.

\section{References}

[1] Ruggeri, Z.M, Platelets in atherothrombosis, Nature Med., 8:1227-1234, 2002.

[2] Mendolicchio, G.L. \& Ruggeri, Z.M., New perspectives on von Willebrand factor functions in hemostasis and thrombosis, Semin. Hematol., 42:5-14, 2005.

[3] Zhu, C., Kinetics and mechanics of cell adhesion, J. Biomech., 33:23-33, 2000.

[4] Savage, B., Almus-Jacobs, F. \& Ruggeri, Z.M., Specific synergy of multiple substrate-receptor interactions in platelet thrombus formation under flow, Cell, 94:657-666, 1998.

[5] Doggett, T.A., Girdhar, G., Lawshe, A., Schmidtke, D.W., Laurenzi, I.J., Diamond, S.L. \& Diacovo, T.G., Selectin-like kinetics and biomechanics promote rapid platelet adhesion in flow: the GP Ib $\alpha-\mathrm{vWF}$ tether bond, Biophys. J., 83:194-205, 2002.

[6] Kumar, R.A., Dong, J., Thaggard, J.A., Cruz, M.A., Lopez, J.A. \& McIntire, L.V., Kinetics of GPIb $\alpha$-vWF-A1 tether bond under flow: effect of GPIb $\alpha$ mutations on the association and dissociation rates, Biophys. J., 85:4099-4109, 2003.

[7] Pierres, A., Benoliel, A. M. \&. Bongrand, P., Use of a laminar flow chamber to study the rate of bond formation and dissociation between surface-bound adhesion molecules: effect of applied force and distance between surfaces, Faraday Discuss., 111:321-330, 1998. 
[8] Mazzucato, M., Pradella. P., Cozzi. M.R., De Marco. L. \& Ruggeri Z.M., Sequential cytoplasmic calcium signals in a 2-stage platelet activation process induced by the glycoprotein Ib $\alpha$ mechanoreceptor, Blood, 100:2793-2800, 2002.

[9] Machin, M., Santomaso, A. Cozzi, M.R., Battiston, M., Mazzucato, M. De Marco, L. \& Canu P., Characterization of platelet adhesion under flow using microscopic image sequence analysis, Int. J. Artif. Organs, in press, 2005.

[10] MATLAB, The MathWorks, Inc., version 7 R14 SP1, 2004.

[11] Machin, M., Santomaso, A., Mazzucato, M., De Marco, L. \& Canu, P. Single particle tracking across sequences of microscopical images: application to platelet adhesion under flow conditions, Ann. Biomed. Eng., submitted, 2005.

[12] Bell, G.I, Models for the specific adhesion of cells to cells, Science, 200:618-627, 1978.

[13] Aarts, P.A.M.M., van den Broek, S.A.T., Prins, G.W., Kuiken, G.D.C., Sixma, J.J. \& Heethaar, R.M., Blood platelets are concentrated near the wall and red blood cells, in the center of flowing blood, Arterioscler., 8:819-824, 1988. 